\title{
STRONG PREINJECTIVE PARTITIONS AND REPRESENTATION TYPE OF ARTINIAN RINGS
}

\author{
BIRGE ZIMMERMANN-HUISGEN
}

(Communicated by Donald S. Passman)

\begin{abstract}
It is shown that for every ring of left pure global dimension zero (i.e., for every ring all of whose left modules are direct sums of finitely generated modules), the finitely generated left modules can be grouped to a unique "strong preinjective partition" while the finitely presented right modules possess a "strong preprojective partition"; these strong partitions are upgraded versions of the partitions introduced by Auslander and Smalø for Artin algebras. One direct consequence is that a ring of left pure global dimension zero has finite representation type if and only if there exist sufficiently many almost split maps among its finitely generated left modules. This provides a very elementary proof for Auslander's theorem saying that for Artin algebras vanishing of the left pure global dimension is equivalent to finiteness of the representation type.
\end{abstract}

\section{INTRODUCTION}

Over a ring of vanishing left pure global dimension (equivalently, a ring all of whose left modules are direct sums of finitely gerierated modules) there are, for each positive integer $n$, only finitely many isomorphism types of left modules of length bounded by $n$ (see [21, 22]). The purpose of the present note is to provide a better understanding of the category of finitely generated modules for such a ring (towards the ultimate goal of establishing or excluding the existence of a candidate which fails to be of finite representation type). For a brief outline of the history of the enterprise to characterize the rings of left pure global dimension zero see the introduction to [21].

More specifically, we will show that, given a ring $R$ of left pure global dimension zero, each family of finitely generated left $R$-modules has a "strong preinjective partition", and each family of finitely presented right $R$-modules has a "strong preprojective partition." These are upscaled versions of the preinjective resp. preprojective partitions introduced and described for Artin algebras by Auslander and Smalø in [6]. More explicitly, Auslander and Smalø showed that any transversal $\mathscr{C}$ of the finitely generated indecomposable modules over an Artin algebra contains disjoint finite subsets $\mathscr{C}_{\alpha}, \alpha<\omega$, such that for each

Received by the editors October 24, 1988 and, in revised form, July 27, 1989.

1980 Mathematics Subject Classification (1985 Revision). Primary 16A35, 16A46, 16A48.

This work was partially supported by a grant from the National Science Foundation. 
$\alpha$ the set $\mathscr{C}_{\alpha}$ is a minimal cogenerating set for $\mathscr{C} \backslash \bigcup_{\beta<\alpha} \mathscr{C}_{\beta}$ (or, equivalently, such that each embedding of a module from $\mathscr{C}_{\alpha}$ into a finite direct sum of modules in $\mathscr{C} \backslash \bigcup_{\beta<\alpha} \mathscr{C}_{\beta}$ splits). If $\mathscr{C}^{\prime}=\mathscr{C} \backslash \bigcup_{\alpha<\omega} \mathscr{C}_{\alpha}$, the (unique) partition $\mathscr{C}=\left(\bigcup_{\alpha<\omega} \mathscr{C}_{\alpha}\right) \cup \mathscr{C}^{\prime}$ was dubbed the preinjective partition for $\mathscr{C}$. Preprojective partitions over Artin algebras were defined and established dually. For certain classes $\mathscr{C}$ of finitely generated modules (over rings which are not Artin algebras), this idea can be pushed beyond the first limit ordinal, namely, the game can be continued for the class $\mathscr{C} \backslash \bigcup_{\alpha<\omega} \mathscr{C}_{\alpha}$; the resulting partition of $\mathscr{C}$ will then be called a strong preinjective partition, and the least ordinal $\mu$ with $\mathscr{C}_{\mu}=\varnothing$ will be called its length. We can now state the most important case of our existence theorem for strong preinjective partitions with more precision:

Theorem A. If $R$ has left pure global dimension zero, then the family of all finitely generated indecomposable left $R$-modules has a unique strong preinjective partition of countable length; this length is neither a limit ordinal nor the successor of a limit ordinal. Analogously, the family of all finitely presented indecomposable right $R$-modules possesses a unique strong preprojective partition of countable length; provided that $R$ is right artinian and has a Morita duality on the right, the same additional restrictions as above apply to this length.

We will use this result to give a short and very elementary proof for Auslander's celebrated theorem asserting that every Artin algebra of left pure global dimension zero has finite representation type. In fact, it follows effortlessly from the existence of strong preinjective partitions that if there are left almost split monomorphisms originating from arbitrary noninjective finitely generated indecomposable left $R$-modules, left pure global dimension zero always implies finite representation type. This result can also be obtained from Auslander's work (combine [2] with [1]); note, moreover, that on the other hand, finite representation type implies the existence of the almost split maps we require (see [1]).

Next we will narrow down the auxiliary condition needed for our bridge from left pure global dimension zero to finite representation type to a "pointwise" condition, that is, one that involves only a single finitely generated left $R$-module at a time. Given any indecomposable finitely generated left $R$-module $A$ with endomorphism ring $S$, denote by $A^{\#}$ the right $R$-module $\operatorname{Hom}_{S}(A, V)$, where $V$ is the injective envelope of the right $S$-module $S / \operatorname{rad} S$.

Corollary $\mathrm{C}$. The following statements are equivalent for any ring $R$ :

(1) $R$ is of finite representation type.

(2) $R$ has left pure global dimension zero, and for each finitely generated indecomposable left $R$-module $A$ the right $R$-module $A^{\#}$ is finitely presented.

It is readily checked that for every finitely generated indecomposable left $R$ module $A$ the right $R$-module $A^{\#}$ is algebraically compact and has in turn a 
local endomorphism ring. For the case where $R$ has left pure global dimension zero without being representation-finite, Corollary $C$ thus yields algebraically compact indecomposable right $R$-modules which fail to be finitely presented. (In this context note that if there exists a ring of vanishing left pure global dimension which is not of finite representation type, then there exists a twosided artinian ring with these properties; see Theorem 1.)

Terminology and prerequisites. Let $R$ be any ring, $\mathscr{M}$ a full subcategory of the category $R$-Mod of left $R$-modules; most often we will consider $\mathscr{M}$ equal to $R$-mod, the full subcategory of all finitely generated left $R$-modules. (Our notation for the corresponding categories of right $R$-modules will be symmetric, with $R$ placed on the right-hand side.) Following Auslander and Reiten [5], we call a nonsplit monomorphism $f: A \rightarrow B$ in $R$-mod with $A, B$ in $\mathscr{M}$ left almost split in $\mathscr{M}$ if each homomorphism $A \rightarrow D$ in $\mathscr{M}$ which is not a split monomorphism factors through $f$; right almost split epimorphisms in $\mathscr{M}$ are defined dually. Finally, an exact sequence $0 \rightarrow A \rightarrow B \rightarrow C \rightarrow 0$ in $R$-mod with $A, B, C$ in $\mathscr{M}$ is said to be almost split in $\mathscr{M}$ if the monomorphism $A \rightarrow B$ is left almost split in $\mathscr{M}$ and the epimorphism $B \rightarrow C$ is right almost split in $\mathscr{M}$.

Call a left $R$-module $M$ with endomorphism ring $S$ strong if $M$ is finitely generated as an $R$-module, $S$ is local, and the radical $\operatorname{rad}_{S}(M)$ of $M$ viewed as a right $S$-module is small in $M$. The latter condition is clearly satisfied if $M$ is finitely generated over $S$, or if $S$ is left perfect, for instance. Moreover, call $M$ strongly decomposable if $M$ is a finite direct sum of strong modules. Clearly, every module of finite length is strongly decomposable (the condition concerning the radical is satisfied because the endomorphism ring is semiprimary in that case), and so is every finitely presented right module over a ring of left pure global dimension zero (the latter example will be explained below).

For a family $\mathscr{C}$ of left $R$-modules denote by Ind $\mathscr{C}$ any family of representatives for the isomorphism types of the indecomposable direct summands of modules in $\mathscr{C}$; in case $\mathscr{C}$ is the family of all finitely presented left $R$-modules, we will also write $R$-Ind for Ind $\mathscr{C}$. Moreover, $\operatorname{Add} \mathscr{C}$ will denote the full subcategory of $R$-mod consisting of all left $R$-modules isomorphic to direct summands of finite direct sums of members of $\mathscr{C}$. We say that $\mathscr{C}$ has left almost split maps if for each noninjective module $A \in \operatorname{Ind} \mathscr{C}$ there exists a left almost split monomorphism $f: A \rightarrow B$ in $R$-mod with $B \in \operatorname{Add} \mathscr{C}$.

Given families $\mathscr{C}, \mathscr{G}$ of finitely generated left $R$-modules, we call $\mathscr{G}$ a cogenerating set for $\mathscr{C}$ if each module in $\mathscr{C}$ can be embedded into a member of Add $\mathscr{G}$. Dually, we say that $\mathscr{G}$ generates $\mathscr{C}$ if each module in $\mathscr{C}$ is an epimorphic image of a member of Add $\mathscr{G}$. Furthermore, for left $R$-modules $A$ and $B$ we denote by $\mathscr{R e}_{B}(A)$ the reject of $B$ in $A$, i.e., $\mathscr{R e}_{B}(A)$ is the intersection of all kernels of homomorphisms from $A$ to $B$; the reject in $A$ of a family $\mathscr{C}$ of modules is the intersection of all the rejects $\mathscr{R e}_{B}(A), B \in \mathscr{C}$. Finally, the trace of $B$ in $A$, denoted $\mathscr{T}_{r_{B}}(A)$, is the sum of all images of homomorphisms from $B$ to $A$. 
Three devices for shifting information from left modules to right modules and vice versa will be applied:

(a) Morita duality. We call $R$ left Morita if $R$ admits a Morita-duality $R-\bmod \rightarrow \bmod -T$ for some ring $T$ ( $T$ will then be right Morita). As is well known (see e.g. [16, Theorems 3.7, 3.8]), $R$ is left Morita precisely if $R$ is left artinian and the minimal injective cogenerator for $R$-Mod is finitely generated; in that case every finitely generated injective cogenerator $M$ for $R$-Mod induces a Morita duality $\operatorname{Hom}_{R}(-, M): R-\bmod \rightarrow \bmod -\operatorname{End}_{R}(M)$.

Theorem 1 (Hullinger-Simson duality principle; see [11, Theorem 7] and [15]). Suppose that $R$ has left pure global dimension zero. Then there exists a ring $T$ of left pure global dimension zero which is artinian and Morita on both sides and has the same representation type as $R$.

More precisely: If $E$ is the minimal injective cogenerator for $R$-Mod and $T=\operatorname{End}_{R}(E)$, then $T$ is two-sided artinian, two-sided Morita, and again has left pure global dimension zero; moreover, the functor $\operatorname{Hom}_{R}(-, E)$ induces a Morita duality $R$-mod $\rightarrow \bmod -T$ (and thus induces a bijection between the isomorphism classes of indecomposable finitely generated left $R$-modules and the classes of indecomposable finitely generated right $T$-modules).

Proof. Suppose that $R$ is as above. Then $R$ is left artinian by [8, Theorem 4.4], and the minimal injective cogenerator $E$ for $R$-Mod, being a finite direct sum of indecomposable left $R$-modules, is finitely generated. Thus there exists a Morita-duality $R$-mod $\rightarrow \bmod -T$, where $T=\operatorname{End}_{R}(E)$. Then $T$ is clearly right artinian and right Morita. On the other hand, by [11, Theorem 7], $T$ in turn has left pure global dimension zero. As we just pointed out, $T$ is thus also left artinian and left Morita.

(b) The Auslander-Bridger transpose. For the basics on the Auslander-Bridger transpose Tr: $R$-Ind $\rightarrow$ Ind $-R$ resp. Tr:Ind $-R \rightarrow R$-Ind for a semiperfect ring $R$ we refer the reader to [4] or to [21, $2($ a)] for a brief summary of what will be needed here.

(c) The assignment $A \rightarrow A^{\#}$ as introduced above.

Remark 2 (see [18, proof of Satz 1]). For every finitely generated indecomposable left $R$-module $A$ the endomorphism ring of the right $R$-module $A^{\#}=$ $\operatorname{Hom}_{S}(A, V)$ is isomorphic to that of the $S$-module $V$. In particular, the right $R$-module $A^{\#}$ is in turn indecomposable.

Proof. Consider the following sequence of isomorphisms of abelian groups:

$$
\begin{aligned}
\operatorname{Hom}_{S}(V, V) & \cong \operatorname{Hom}_{S}\left(\operatorname{Hom}_{S}\left(\operatorname{Hom}_{R}(A, A), V\right), V\right) \\
& \cong \operatorname{Hom}_{S}\left(\operatorname{Hom}_{S}(A, V) \otimes_{R} A, V\right) \\
& \cong \operatorname{Hom}_{R}\left(\operatorname{Hom}_{S}(A, V), \operatorname{Hom}_{S}(A, V)\right) .
\end{aligned}
$$


Note that the canonical map $\operatorname{Hom}_{S}(A, V) \otimes_{R} A \rightarrow \operatorname{Hom}_{S}\left(\operatorname{Hom}_{R}(A, A), V\right)$ is bijective by [7, Chap. I, $\S 2$, Exercise 14], and observe that the composition is the map $\operatorname{Hom}_{S}(V, V) \rightarrow \operatorname{Hom}_{R}\left(A^{\#}, A^{\#}\right)$ which sends $f$ to $(g \mapsto f \cdot g)$. Thus the latter two rings are isomorphic.

\section{STRONG PREINJECTIVE PARTITIONS AND STRONG PREPROJECTIVE PARTITIONS}

The following "strong preinjective (preprojective) partitions" of families of finitely generated modules refine, existence provided, the preinjective (preprojective) partitions introduced by Auslander and Smalø [6]. Existence, however, occurs rather rarely, which is the pivotal point of this paper.

Definition 3. Let $\mathscr{C}$ be a family of finitely generated (left) $R$-modules. We say that $\mathscr{C}$ has a strong preinjective partition if there exist an ordinal number $\tau$ and a partition Ind $\mathscr{C}=\bigcup_{\alpha<\tau} \mathscr{C}_{\alpha}$ with the following properties:

(i) $\mathscr{C}_{\alpha} \cap \mathscr{C}_{\beta}=\varnothing$ whenever $\alpha, \beta$ are distinct ordinals below $\tau$;

(ii) each $\mathscr{C}_{\alpha}$ is finite;

(iii) for each ordinal $\alpha$, the set $\mathscr{C}_{\alpha}$ is a minimal cogenerating set for $\bigcup_{\beta \geq \alpha} \mathscr{C}_{\beta}$.

The least ordinal $\mu$ with $\mathscr{C}_{\mu}=\varnothing$ is called the length of the partition.

Strong preprojective partitions are defined dually.

Clearly, given a strong preinjective partition Ind $\mathscr{C}=\bigcup_{\alpha<\tau} \mathscr{C}_{\alpha}$ such that $\mathscr{C}_{\beta}=\varnothing$, we have $\mathscr{C}_{\gamma}=\varnothing$ for all $\gamma \geq \beta$; in particular, Ind $\mathscr{C}^{\alpha<\tau}$ will be finite if $\mathscr{C}_{\beta}=\varnothing$ for some $\beta<\omega$.

The following theorem, due to Auslander and Smalø, is the key to effective exploitation of strong preinjective partitions. Since it was originally established in a slightly more specialized context, we include the short argument for the reader's convenience.

Theorem 4 (Auslander and Smalø [6, Theorem 2.3]). Let $\mathscr{C}$ be a family of strongly decomposable modules.

(1) Suppose that $\mathscr{C}$ has a strong preinjective partition, say Ind $\mathscr{C}=\bigcup_{\alpha<\tau} \mathscr{C}$. If we set $\mathscr{D}_{\alpha}=\bigcup_{\beta \geq \alpha} \mathscr{C}_{\beta}$, then each $\mathscr{C}_{\alpha}$ consists precisely of those modules $C \in \mathscr{D}_{\alpha}$ for which every monomorphism $C \rightarrow M$ with $M \in$ Add $\mathscr{D}_{\alpha}$ splits.

(2) Suppose that $\mathscr{C}$ has a strong preprojective partition, say Ind $\mathscr{C}=$ $\bigcup_{\alpha<\tau} \mathscr{C}_{\alpha}^{*}$. If we set $\mathscr{D}_{\alpha}^{*}=\bigcup_{\beta \geq \alpha} \mathscr{C}_{\beta}^{*}$, then each $\mathscr{C}_{\alpha}^{*}$ consists precisely of those modules $C \in \mathscr{D}_{\alpha}^{*}$ for which every epimorphism $M \rightarrow C$ with $M \in \operatorname{Add} \mathscr{D}_{\alpha}^{*}$ splits.

Proof. We prove (2). It clearly suffices to show that if $\mathscr{C}$ is a family of strong $R$-modules (right $R$-modules say) admitting a minimal generating set $\mathscr{A} \subseteq$ $\mathscr{C}$, then $\mathscr{A}$ coincides with the set $\mathscr{A}^{\prime}$ of those modules $A \in \mathscr{C}$ for which 
every epimorphism $M \rightarrow A$ with $M \in \operatorname{Add} \mathscr{C}$ splits. Clearly, $\mathscr{A}^{\prime} \subseteq \mathscr{A}$. For the other inclusion, pick $A \in \mathscr{A}$ and let $f: M \rightarrow A$ be an epimorphism with $M \in \operatorname{Add} \mathscr{C}$; since $\mathscr{A}$ generates Add $\mathscr{C}$, we can find an epimorphism $g:\left(\bigoplus_{i=1, \ldots, m} B_{i}\right) \oplus A^{n} \rightarrow M$ with $B_{1}, \ldots, B_{m} \in \mathscr{A}-\{A\}$. If we can show that the epimorphism $h=f \cdot g$ splits, then the original epimorphism $f$ will split as well.

In order to show that $h$ splits, set $B=\bigoplus_{i=1, \ldots, m} B_{i}$ and $S=\operatorname{End}_{R}(A)$; moreover, let $h_{1}, \ldots, h_{n}$ be the restrictions of $h$ to the various copies of $A$. If one of the $h_{i}$ is an isomorphism, then $h$ clearly splits. Otherwise, all $h_{i}$ belong to $\operatorname{rad}(S)$ due to the locality of $S$. Since $\operatorname{rad}_{S}(A)$ is $S$-small in $A$ by hypothesis and $\mathscr{T}_{\mu_{B}}(A)$ is an $S$-submodule of $A$, we obtain $A=\mathscr{T}_{\mu_{B}}(A)$ from the equality $A=h_{1} A+\cdots+h_{m} A+\mathscr{T}_{n_{B}}(A)$. But this means that $A$ can be generated by $B_{1}, \ldots, B_{m}$ (strong modules are finitely generated), which contradicts the minimality of $\mathscr{A}$ as a generating set for $\mathscr{C}$.

Proposition 5. Let $\mathscr{C}=\left(M_{i}\right)_{i \in I}$ be a family of finitely generated right $R$-modules whose direct sum $\bigoplus_{i \in I} M_{i}$ is noetherian over its endomorphism ring. Then each subfamily of $\operatorname{Add} \mathscr{C}$ has a strong preprojective partition. If the $M_{i}$ are strongly decomposable, this partition is unique (namely as described in Theorem 4(2)).

Proof. It is clearly harmless to assume that $\mathscr{C}$ consists of indecomposable modules. Moreover, it is obvious that, given a module $M$ which is noetherian over its endomorphism ring, every direct summand of $M$ inherits this property. As a consequence, every direct sum extending over a subfamily of $\mathscr{C}$ is again noetherian over its endomorphism ring. For the existence statement it is therefore enough to show that $\mathscr{C}$ contains a finite subfamily which generates $\mathscr{C}$; since every such finite generating set will contain a minimal one, an obvious transfinite induction will then complete the proof. Set $M=\bigoplus_{i \in I} M_{i}$ and $S=\operatorname{End}_{R}(M)$. Since $M$ is finitely generated over $S$, there exist $i_{1}, \ldots, i_{n} \in I$ such that $M$, as an $S$-module, is generated by $M_{i_{1}} \oplus \cdots \oplus M_{i_{n}}$. But then the set $\left\{M_{i_{1}}, \ldots, M_{i_{n}}\right\}$ clearly generates $\mathscr{C}$. The uniqueness statement follows from Theorem 4.

The crucial fact is that if $R$ has vanishing left pure global dimension, the hypothesis of Proposition 5 is satisfied for any family of finitely presented right $R$-modules by [21, Theorem 9].

Theorem A (first part). Suppose that $R$ has left pure global dimension zero. Then

(1) every family $\mathscr{C}$ of finitely generated left $R$-modules has a unique strong preinjective partition of countable length $\mu$, say Ind $\mathscr{C}=\bigcup_{\alpha<\mu} \mathscr{C}_{\alpha}$, where each of the components $\mathscr{C}_{\alpha}$ is as described in Theorem 4(1); moreover,

(2) every family $\mathscr{C}^{*}$ of finitely presented right $R$-modules has a unique strong preprojective partition of countable length with components as described in Theorem 4(2). 
Proof. We start by showing (2).

Due to the fact that each direct sum of finitely presented right $R$-modules is noetherian over its endomorphism ring [21, loc. cit.], the existence of a strong preprojective partition for any family $\mathscr{C}^{*}$ of finitely presented right modules follows from Proposition 5. Since by [21, Corollary 10] the class of all isomorphism types of finitely presented right $R$-modules is countable, the length $\nu$ of any such partition is countable.

The description of the components $\mathscr{C}_{\alpha}^{*}$ will follow from Theorem 4 once we have checked that each finitely presented indecomposable right $R$-module $M$ is strong (by means of the Auslander-Bridger transpose, it can easily be verified that each finitely presented right $R$-module is a direct sum of indecomposable components). To show that $M$ has a local endomorphism ring, we may clearly assume that $M$ is nonprojective. Then $\operatorname{Tr} M$ has a local endomorphism ring, and

$$
\operatorname{End}_{R}(M) / P(M) \cong \operatorname{End}_{R}(\operatorname{Tr} M) / P(\operatorname{Tr} M),
$$

where $P(M)$ resp. $P(\operatorname{Tr} M)$ denotes the ideal of those endomorphisms which can be factored through a projective module (and where endomorphisms act opposite the scalars in each case); since clearly $P(M)$ is a nilpotent ideal of $\operatorname{End}_{R}(M)$, the latter ring inherits the property of being local from the factor ring $\operatorname{End}_{R}(M) / P(M)$. The condition concerning the radical of $M$ viewed as a module over its endomorphism ring is satisfied because, as we already noted, each finitely presented right module is neotherian over its endomorphism ring. Thus $M$ is indeed strong.

(1) The dual statements concerning finitely generated left $R$-modules can now be deduced from Theorem 1. Using the notation of that Theorem, let $D: R-\bmod \rightarrow \bmod -T$ be a Morita duality. Given any family $\left(C_{i}\right)_{i \in I}$ of finitely generated left $R$-modules, the family $\left(D\left(C_{i}\right)\right)_{i \in I}$ has a unique strong preprojective partition as we just saw. The mirror image of this partition under $D$ is then a strong preinjective partition of the original family with the desired properties.

If $R$ has left pure global dimension zero, then Theorem A yields in particular a strong preinjective partition (of countable length) for the family $R$-Ind of all isomorphism types of finitely generated indecomposable left $R$-modules, as well as a strong preprojective partition for the family Ind- $R$ of all isomorphism types of finitely presented indecomposable right $R$-modules. While primarily our interest is directed towards conditions which force the length of either one of these partitions to be finite (the latter conditions being alternate ways of saying that $R$ is of finite representation type), we will in the sequel gain some general insights concerning their lengths.

Observation 6 . Let $\mathscr{C}$ be a family of strongly decomposable left modules, $C$ a member of Ind $\mathscr{C}$, and suppose that $C$ can be cogenerated by infinitely many disjoint finite subsets of Ind $\mathscr{C}$. If $\mathscr{C}$ has a strong preinjective partition, say Ind $\mathscr{C}=\bigcup_{\alpha<\tau} \mathscr{C}_{\alpha}$, then $C \in \bigcup_{\alpha \geq \omega} \mathscr{C}_{\alpha}$. 
A special case: Let $R$ be right perfect, and suppose that $\mathscr{C}$ is a family of strongly decomposable left $R$-modules including representatives $S_{1}, \ldots, S_{n}$ of the simple left $R$-modules and endowed with a strong preinjective partition. Then the length of this partition cannot be a limit ordinal; in particular, either Ind $\mathscr{C}$ is finite, or else the strong preinjective partition of $\mathscr{C}$ has length greater than $\omega$.

Proof. Clearly, for any finite ordinal $\alpha$ the finite set $\bigcup_{\beta<\alpha} \mathscr{C}_{\beta}$ has empty intersection with infinitely many of the given disjoint finite subsets of Ind $\mathscr{C}$ cogenerating $C$. In particular, there exists a nonsplit monomorphism $C \rightarrow M$ for some $M \in \operatorname{Add}\left(\bigcup_{\beta \geq \alpha} \mathscr{C}_{\beta}\right)$, which shows that $C \notin \mathscr{C}_{\alpha}$. This proves the first claim.

For the special case, suppose that $R$ is right perfect, $\mathscr{C}$ as specified, and let Ind $\mathscr{C}=\bigcup_{\alpha<\tau} \mathscr{C}$ be the strong preinjective partition. Moreover, let $\nu$ be the least ordinal such that $S_{1}, \ldots, S_{n} \in \bigcup_{\alpha \leq \nu} \mathscr{C}_{\alpha}$. Then $\mathscr{C}_{\nu+1}$ must be empty by Theorem 4 , since every nonsimple indecomposable module $M$ gives rise to a nonsplit embedding $S_{i} \rightarrow M$ for some $i$. Thus the length of the partition is $\nu+1$. The concluding claim is now obvious.

The proof of the following proposition is a rearrangement of ideas of Auslander and Smalø.

Proposition 7. Let $\mathscr{C}$ be a family of left $R$-modules of finite length (or of strongly decomposable modules all of which are noetherian over their endomorphism rings), and suppose that $\operatorname{Add} \mathscr{C}$ is closed under factoring out rejects, i.e., $A / \mathscr{R e}_{B}(A) \in \operatorname{Add} \mathscr{C}$ whenever $A, B \in \operatorname{Add} \mathscr{C}$. Moreover, suppose that Ind $\mathscr{C}=\bigcup_{\alpha<\tau} \mathscr{C}$ is a strong preinjective partition. Then, given any limit ordinal $\gamma<\tau$, no member of $\mathscr{C}_{\gamma}$ occurs as the domain of a left almost split monomorphism in Add $\mathscr{C}$.

In particular, this yields: If $\mathscr{C}$ has left almost split maps, then the length of the partition is at most $\omega$.

Proof (compare proof of Theorem 7.1 in [6]). Under either assumption, all the modules in $\mathscr{C}$ are strongly decomposable. Let $A \in \mathscr{C}_{\gamma}$, where $\gamma$ is a limit ordinal below $\tau$, and assume there is a left almost split monomorphism $f: A \rightarrow X$ with $X \in \operatorname{Add} \mathscr{C}$. Since for each $\alpha<\gamma$ the set $\mathscr{C}_{\alpha}$ cogenerates $A$, we can find a (nonsplit) monomorphism $f_{\alpha}: A \rightarrow M_{\alpha}$ with $M_{\alpha} \in \operatorname{Add} \mathscr{C}_{\alpha}$. Write $X^{\alpha}$ for the reject of $\mathscr{C}_{\alpha}$ in $X$, and observe that $X^{\beta} \subseteq X^{\alpha}$ for $\beta<\alpha<\gamma$. All the $X^{\alpha}$ being $R$ - $S$-submodules of $X$, where $S$ denotes the endomorphism ring of $X$, this chain will become stationary from a certain $\beta<\gamma$ on (for either choice of assumption on the members of $\mathscr{C}$ ). By hypothesis we have $X / X^{\beta} \in \operatorname{Add} \mathscr{C}$. Moreover, since each indecomposable summand $Y$ of $X / X^{\beta}$ is cogenerated by $\mathscr{C}_{\alpha}$ for all $\alpha<\gamma$, we have $Y \in \bigcup_{\alpha \geq \gamma} \mathscr{C}_{\alpha}$ (see proof of Observation 6). Thus $X / X^{\beta} \in \operatorname{Add} \bigcup_{\alpha \geq \gamma} \mathscr{C}_{\alpha}$. Now factor each $f_{\alpha}$ through $f$, say $f_{\alpha}=g_{\alpha} \cdot f$ with $g_{\alpha}: X \rightarrow M_{\alpha}$. For $\beta<\alpha<\gamma$ the map $g_{\alpha}$ is really a map 
$g_{\alpha}: X / X^{\beta} \rightarrow M_{\alpha}$, whence the homomorphism $f^{*}: A \rightarrow X / X^{\beta}$ induced by $f$ is still a monomorphism. Since $f$ does not split, neither does $f^{*}$. But in light of Theorem 4, this contradicts the choice of $A \in \mathscr{C}_{\gamma}$.

Note that both Observation 6 and Proposition 7 have obvious duals pertaining to strong preprojective partitions.

The first statement of the following supplement to Theorem A can also be obtained by combining Auslander's [2, Proposition 2.1] with his ideas in [1]; the specialization to Artin algebras is Theorem A of [2]. Note that to prove Theorem $A^{\prime}(I)$ below, we need only a small portion of the results we developed up to now, which makes this part of Theorem $A^{\prime}$ quite easily accessible. Recall that by saying that $R$-mod has left almost split maps we mean that for every noninjective finitely generated indecomposable left $R$-module $A$ there is a left almost split map in $R$-mod starting in $A$.

Theorem $\mathbf{A}^{\prime}$. Suppose that $R$ has left pure global dimension zero.

(I) If $R$-mod has left almost split maps, then $R$ is of finite representation type. (In particular, every Artin algebra of vanishing left pure global dimension has finite type.)

(II) In general, the length of the strong preinjective partition of $R$-Ind is neither a limit ordinal nor the successor of a limit ordinal. In case $R$ is also right artinian and right Morita, the same is true for the length of the strong preprojective partition of Ind- $R$.

Proof. To prove the first claim, suppose that $R$-mod has left almost split maps. The ring $R$ being left artinian, $\operatorname{Add}(R-\operatorname{Ind})=R-\bmod$ is closed under factor modules. Thus Proposition 7 proves the length $\tau$ of the strong preinjective partition of $R$-Ind to be at most $\omega$. But since the simple left $R$-modules belong to $R$-Ind, either $\tau$ is finite or $\tau>\omega$ by Observation 6 . Combining these bounds, we see that $\tau$ is finite, meaning that $R$ has finite representation type.

To establish the first part of (II), let $R$-Ind $=\bigcup_{\alpha<\mu} \mathscr{C}_{\alpha}$ be the strong preinjective partition of $R$-Ind, and $\mu$ its length. From Observation 6 we know that $\mu$ is a successor ordinal, say $\mu=\nu+1$. Since moreover every nonsimple finitely generated indecomposable $R$-module is a nonsplit extension of a simple module, $\mathscr{C}_{\nu}$ contains a simple left $R$-module, $A$ say. Clearly, we may assume that $R$ is not semisimple, which is to say that $\nu>0$; in particular, $A$ is then noninjective. Note however that $A$ is algebraically compact over $R$ by hypothesis. If $S$ denotes the division ring of endomorphisms of $A$, then $A$ is finite dimensional over $S$, and $A^{\#}=\operatorname{Hom}_{S}(A, S)$ is a cyclic right $R$-module by the Jacobson density theorem. Thus [18, Satz 6(1)] guarantees the existence of an almost split sequence $0 \rightarrow A \rightarrow B \rightarrow \operatorname{Tr}\left(A^{\#}\right) \rightarrow 0$ in $R$-mod. But now Proposition 7 shows that $\nu$ cannot be a limit ordinal, which completes the proof of the claim for $R$-Ind. 
Next suppose that $R$ is also right artinian and right Morita, and let $D^{\prime}$ : $\bmod -R \rightarrow R^{\prime}$ - mod be a Morita duality (the inverse of $D^{\prime}$ is also denoted by $\left.D^{\prime}\right)$. Since $R$ has vanishing left pure global dimension, the family $\mathscr{F}$ of all homomorphisms between finitely presented indecomposable right $R$-modules is artinian (see e.g. [21, Corollary 5]), meaning that for each family $\left(f_{i}\right)_{i \in \mathbf{N}}$ of nonisomorphisms in $\mathscr{F}$ such that the codomain of $f_{i+1}$ equals the domain of $f_{i}$ there exists an index $m$ such that $f_{1} \cdots f_{m}=0$. Flipping sides via the duality $D^{\prime}$, we infer that the family of all homomorphisms between finitely generated indecomposable left $R^{\prime}$-modules is noetherian (dual of "artinian" as introduced above). But by [9, Theorem] this shows that $R^{\prime}$ in turn has left pure global dimension zero. Applying the previous paragraph to $R^{\prime}$-Ind, we obtain that the length of the strong preinjective partition of $R^{\prime}$-Ind is neither a limit ordinal nor the successor of a limit ordinal. Shifting this information back to Ind $-R$ by means of $D^{\prime}$ now yields the last claim.

Remark 8. As was proved by Auslander [1, duals of Theorems 2.10 and 3.1], rings of finite representation type do have the property that all their noninjective indecomposable left (right) modules occur as first terms of almost split sequences in $R$-mod $(\bmod -R)$, and all their nonprojective indecomposable modules occur as last terms of such sequences. Thus the hypothesis concerning left almost split maps in the first part of Theorem $\mathrm{A}^{\prime}$ is also necessary for the conclusion.

Remark 9. Suppose that $R$ is left artinian and $C$ an arbitrary nonprojective finitely generated indecomposable left $R$-module. By [3, Theorem 3.9] there exists an almost split sequence $0 \rightarrow A \rightarrow B \rightarrow C \rightarrow 0$ in $R$-Mod; in particular, $A$ is indecomposable. Consequently, if $R$ has vanishing left pure global dimension, then $A$ is finitely generated, and the above sequence belongs to $R$-mod . Moreover, since almost split sequences are completely determined (up to isomorphism) by their left-hand terms, we thus obtain an infinite family $\mathscr{C}$ of finitely generated indecomposable left $R$-modules which occur as domains of left almost split maps in $R$-mod, provided that $R$ has left pure global dimension zero but infinite representation type. Furthermore, Theorem A tells us that $\mathscr{C}$ has a strong preinjective partition. It thus appears promising to apply the method of Theorem $\mathrm{A}^{\prime}(\mathrm{I})$ to obtain a contradiction in certain cases; however, the crux now is the necessity to check the closedness condition for $\operatorname{Add} \mathscr{C}$ in Proposition 7.

In analyzing the proof of Proposition 7 and its bearing on the first part of Theorem $\mathrm{A}^{\prime}$, one notices that we require far less than the existence of left almost split maps in $R$-mod for our argument. In fact, suppose that $R$ has left pure global dimension zero, let $R$-Ind $=\bigcup_{\alpha<\tau} \mathscr{E} \alpha$ be the strong preinjective partition of $R$-Ind, and follow Auslander and Smalø in calling the members of $\bigcup_{\alpha<\omega} \mathscr{C}_{\alpha}$ the preinjective left $R$-modules. Given a module $A \in \mathscr{C}_{\omega}$, we have (by definition) infinitely many nonsplit embeddings $f_{n}: A \rightarrow \prod_{I(n)} A_{i}$, where the sets $\left\{A_{i}: i \in I(n)\right\}$ are pairwise disjoint finite sets of preinjective modules. 
It is straightforward to check that the existence of an almost split map $A \rightarrow *$ entails nonsplitness of the diagonal map $f=\left(f_{n}\right): A \rightarrow \prod_{I} A_{i}$, where $I$ is the union of the sets $I(n)$ (this implication makes no use of our hypothesis "left pure $\operatorname{gl} \operatorname{dim} R=0$ "). On the other hand, all we need in order to arrive at a contradiction is nonsplitness of $f$. Indeed, assume nonsplitness of this map, write $\prod_{I} A_{i}=\bigoplus_{J} B_{j}$ with finitely generated summands $B_{j}$ by hypothesis, and pick a finite subsum $B$ of the $B_{j}$ which contains the image of $f$. If we let $g: A \rightarrow B$ be the composition of $f$ with some projection $\bigoplus_{J} B_{j} \rightarrow B$, then $g$ is not split, and all the maps $f_{n}$ factor through $g$. Thus $g$ will serve the same purpose as a left almost split map $A \rightarrow *$ in our argument for part (I) of Theorem $\mathrm{A}^{\prime}$. As a corollary to the proof of Theorem $\mathrm{A}^{\prime}$ and Proposition 7 we thus record the following sharpening of Auslander's [2, Proposition 2.4]:

Corollary B. The following are equivalent for a ring $R$ :

(1) $R$ has finite representation type.

(2) $R$ has left pure global dimension zero, and each indecomposable direct summand of a direct product of preinjective left $R$-modules is preinjective.

A combination of Theorem $A^{\prime}$ with well-known existence theorems for almost split sequences will next allow us to replace the hypothesis of part (I) of Theorem $A^{\prime}$ by a condition which deals with one finitely generated left module at a time. Recall that, given a finitely generated indecomposable left $R$-module $A$ with endomorphism ring $S$, we denote by $A^{\#}$ the right $R$-module $\operatorname{Hom}_{S}(A, V)$, where $V$ is the injective envelope of $S / \operatorname{rad} S$.

Corollary C. The following statements are equivalent for a ring $R$ :

(1) $R$ has finite representation type.

(2) $R$ has left pure global dimension zero, and for each noninjective finitely generated indecomposable left $R$-module $A$, the right $R$-module $A^{\#}$ is finitely presented.

Proof. For $(1) \Rightarrow(2)$ use the facts that a ring of finite type has left and right pure global dimension zero [14] and that $A^{\#}$ is indecomposable (Remark 2).

(2) $\Rightarrow(1)$. Let $A$ be any noninjective finitely generated indecomposable left $R$-module. Since $A$ is algebraically compact, [18, Satz 6(1)] ensures the exisience of an almost split sequence $0 \rightarrow A \rightarrow B \rightarrow \operatorname{Tr}\left(A^{\#}\right) \rightarrow 0$ in $R$-mod; in particular, there is a left almost split map starting in $A$. Now apply Theorem $\mathrm{A}^{\prime}(\mathbf{I})$.

By Theorem 1 we know that if there exists a ring of vanishing left pure global dimension which has infinite representation type, then there exists a twosided artinian ring with the same properties. Corollary D gives some additional information on this pathology. 
Corollary D. If $R$ is two-sided artinian and has left pure global dimension zero, then either $R$ is of finite representation type, or else $R$ admits indecomposable algebraically compact right modules which fail to be finitely generated.

Proof. Suppose that $R$ satisfies the hypotheses while being of infinite type, and, invoking Corollary $\mathrm{C}$, pick a finitely generated indecomposable left $R$-module $A$ such that the right $R$-module $A^{\#}$ is not finitely presented, that is, not finitely generated. By Remark $2, A^{\#}$ is indecomposable. To see that $A^{\#}$ is algebraically compact, it suffices to observe that $\operatorname{Hom}_{S}(X, Y)$ is algebraically compact as an $R$-module whenever $X$ is an $R$-S-bimodule and $Y$ an algebraically compact $S$-module (use the adjointness of hom-functor and tensorproduct to verify this).

To conclude, we point out a "local" version of Theorem A'(I). In [21, Corollary 12] it was shown that if $R$ is an Artin algebra and $\mathscr{C}$ a family of finitely generated indecomposable $R$-modules whose direct sum is $\Sigma$-algebraically compact over $R$, then for every positive integer $n$ the family $\mathscr{C}$ contains only finitely many isomorphism classes of modules of length bounded by $n$. Using our present methods and specializing to classes $\mathscr{C}$ for which $\operatorname{Add} \mathscr{C}$ is closed under submodules, we can strengthen the latter conclusion considerably.

Corollary $\mathbf{E}$ (to the proof of Theorem $\mathrm{A}^{\prime}$ ). Let $\mathscr{C}$ be a family of finitely generated indecomposable left modules over an Artin algebra $R$ such that Add $\mathscr{C}$ is closed under submodules. Then the following statements are equivalent:

(1) Each direct product of modules in $\mathscr{C}$ is a direct sum of finitely generated modules.

$\left(1^{\prime}\right)$ Each direct product of modules in $\mathscr{C}$ is a direct sum of modules with local endomorphism rings.

(2) The direct sum of the modules in $\mathscr{C}$ is $\sum$-algebraically compact.

(3) $\mathscr{C}$ contains only finitely many pairwise nonisomorphic modules.

Proof. Let $K$ be the center of $R$ and $E$ the injective envelope of $K / \operatorname{rad} K$; by $\mathscr{D}$ denote the functor $\operatorname{Hom}_{K}(-, E)$ which restricts to a Morita self-duality of $R$. Moreover, let $P$ be the direct product of the modules in $\mathscr{C}$ and $Q$ their direct sum.

$(1) \Rightarrow\left(1^{\prime}\right)$ is trivial.

$\left(1^{\prime}\right) \Rightarrow(2)$. By hypothesis, $P^{\mathbf{N}}$ is a direct sum of modules with local endomorphism rings, whence $P$ is $\sum$-algebraically compact by [19, Theorem A]. But by $[17$, Folgerung 3.4$]$ the direct product of the modules in $\mathscr{C}$ is $\sum$-algebraically compact if and only if the direct sum is.

$(2) \Rightarrow(3)$. Suppose that (2) holds, which means that $Q$ has the descending chain condition on matrix subgroups (see [10, Théorème 1.3] or [17, Folgerung 3.4]; moreover, see [20] or [21, $\$ 2$ (b)] for an introduction to matrix subgroups). Using [21, Proposition 3], we infer that the right $R$-module $\mathscr{D}(Q)$ has the ascending chain condition for finite matrix subgroups. But if $Q=\bigoplus_{i \in I} C_{i}$ with $C_{i}$ in $\mathscr{C}$, then $\mathscr{D}(Q)=\prod_{i \in I} \mathscr{D}\left(C_{i}\right)$, and since passage to finite matrix subgroups is a process which commutes with direct products and direct sums, 
we conclude that the sum $Q^{*}=\bigoplus_{i \in I} \mathscr{D}\left(C_{i}\right)$ also satisfies the ascending chain condition for finite matrix subgroups. By [21, Observation 8] we obtain further that $Q^{*}$ is noetherian over its endomorphism ring, whence by Proposition 5 above the family $\mathscr{C}^{*}=\left(\mathscr{D}\left(C_{i}\right)\right)_{i \in I}$ has a strong preprojective partition. Consequently, $\mathscr{C}$ has a strong preinjective partition, say Ind $\mathscr{C}=\bigcup_{\alpha<\tau} \mathscr{C}_{\alpha}$, where $\tau$ is the length of the partition (note that Ind $\mathscr{C}$ is just a transversal of $\mathscr{C}$ because $\mathscr{C}$ consists of indecomposable modules). We want to show that $\tau$ is finite. Assume the contrary, and note that then $\mathscr{C}_{\omega} \neq \varnothing$ (apply Observation 6 to a simple module that occurs in the socle of infinitely many members of Ind $\mathscr{C}$ ). Let $A \in \mathscr{C}_{\omega}$. By [5, Proposition 4.3] the module $A$ occurs as the left-hand term of an almost split sequence in $R$-mod; in particular, there is a left almost split monomorphism $f: A \rightarrow X$ for some $X \in R$-mod. Using the notation introduced in the proof of Proposition 7 based on $\gamma=\omega$, we observe that $X / X^{\beta}$ belongs to $\operatorname{Add} \mathscr{C}$, because $X / X^{\beta}$ is congenerated by all $\mathscr{C}_{\alpha}$, $\alpha<\omega$, and $\operatorname{Add} \mathscr{C}$ is closed under submodules by hypothesis. We now reach a contradiction just as in the proof of Proposition 7. Thus $\tau$ is finite, which means that the members of $\mathscr{C}$ belong to finitely many isomorphism classes.

(3) $\Rightarrow(1)$. The family Ind $\mathscr{C}$ being finite by hypothesis, it clearly suffices to show that for each $C \in \mathscr{C}$ and any index set I the direct product $C^{I}$ is isomorphic to a direct sum of copies of $C$. Letting $S$ denote the endomorphism ring of $C$, it is clear that $S$ contains $K$ as a subring and is module finite over $K$. By Chase's theorem [8, Theorem 3.3] we deduce that $S^{I}$ is projective as a left $S$-module. But since $S$ is local, the latter implies that $S^{I}$ is free as a left $S$-module by Kaplansky's theorem [12, Theorem 2]; that is, $S^{I} \cong S^{(J)}$ for a suitable index set $J$. Since $C$ has finite length over $S$ because $C$ has finite length over $K,\left[13\right.$, Satz 1] finally yields that $C^{I} \cong C \otimes_{S} S^{I} \cong C \otimes_{S} S^{(J)} \cong C^{(J)}$ as desired.

\section{ACKNOWLEDGMENT}

The author wishes to thank W. Zimmermann for several helpful conversations.

\section{REFERENCES}

1. M. Auslander, Representation theory of Artin algebras II, Comm. Algebra 1 (1974), 269-310.

2. L Large modules over Artin algebras, Algebra, Topology and Categories, Academic Press, New York, 1976.

3. _ Functors and morphisms determined by objects, Representation Theory of Algebras, Proc. Philadelphia Conf., New York, 1978, pp. 1-244.

4. M. Auslander and M. Bridger, Stable module theory, Mem. Amer. Math. Soc., no. 94, Amer. Math. Soc., Providence, RI, 1969.

5. M. Auslander and I. Reiten, Representation theory of Artin algebras III, almost split sequences, Comm. Algebra 3 (1975), 239-294.

6. M. Auslander and S. O. Smalø, Preprojective modules over Artin algebras, J. Algebra 66 (1980), 61-122. 
7. N. Bourbaki, Commutative algebra, Hermann, Paris, 1972.

8. S. U. Chase, Direct products of modules, Trans. Amer. Math. Soc. 97 (1960), 457-473.

9. K. R. Fuller, On rings whose left modules are direct sums of finitely generated modules, Proc. Amer. Math. Soc. 54 (1976), 39-44.

10. L. Gruson and C. U. Jensen, Modules algébriquement compact et foncteurs $\lim ^{(i)}$, C. R. Acad. Sci. Paris Sér. A Math. 276 (1973), 1651-1653.

11. H. Hullinger, Stable equivalence and rings whose modules are a direct sum of finitely generated modules, J. Pure Appl. Algebra 16 (1980), 265-273.

12. I. Kaplansky, Projective modules, Ann. of Math. 68 (1958), 372-377.

13. H. Lenzing, Endlich präsentierbare Moduln, Arch. Math. 20 (1969), 262-266.

14. C. M. Ringel and H. Tachikawa, QF-3 rings, J. Reine Angew. Math. 272 (1975), 49-72.

15. D. Simson, Pure semisimple categories and rings of finite representation type, J. Algebra 48 (1977), 290-296.

16. H. Tachikawa, Quasi-Frobenius rings and genealizations, Lecture Notes in Math., vol. 351, Springer, Berlin, 1973.

17. W. Zimmerman, Rein-injektive direkte Summen von Moduln, Comm. Algebra 5 (1977), 1083-1117.

18. __ Existenz von Auslander-Reiten-Folgen, Arch. Math. 40 (1983), 40-49.

19. B. Zimmermann-Huisgen, Rings whose right modules are direct sums of indecomposable modules, Proc. Amer. Math. Soc. 77 (1979), 191-197.

20. B. Zimmermann-Huisgen and W. Zimmermann, Algebraically compact rings and modules, Math. Z. 161 (1978), 81-93.

21. $\ldots$, On the sparsity of representations of rings of pure global dimension zero, Trans. Amer. Math. Soc. (to appear).

22. M. Prest, Duality and pure-semisimple rings, J. London Math. Soc. 38 (1988), 403-409. 93106

Department of Mathematics, University of California, Santa Barbara, California 